\title{
ON THE OSCILLATORY BEHAVIOR OF SINGULAR STURM-LIOUVILLE EXPANSIONS
}

\author{
BY
}

\author{
J. K. SHAW
}

\begin{abstract}
A singular Sturm-Liouville operator $L y=-\left(P y^{\prime}\right)^{\prime}+Q y$, defined on an interval $\left[0, b^{*}\right)$ of regular points, but singular at $b^{*}$, is considered. Examples are the Airy equation on $[0, \infty)$ and the Legendre equation on $[0,1)$. A mode of oscillation of the successive iterates $f(t),(L f)(t),\left(L^{2} f\right)(t), \ldots$ of a smooth function $f$ is assumed, and the resulting influence on $f$ is studied. The nature of the mode is that for a fixed integer $N>0$, each iterate $\left(L^{k} f\right)(t)$ shall have on $\left(0, b^{*}\right)$ exactly $N$ sign changes which are stable, in a certain sense, as $k$ varies. There is quoted from the literature the main characterization of such functions $f$ which additionally satisfy strong homogeneous endpoint conditions at 0 and $b^{*}$. An extended characterization is obtained by weakening the conditions of $f$ at 0 and $b^{*}$. The homogeneous endpoint conditions are replaced by a summability condition on the values, or limits of values, of $f$ at 0 and $b^{*}$.
\end{abstract}

1. Introduction. Let $L$ be the Sturm-Liouville operator

$$
L y=-\left(P y^{\prime}\right)^{\prime}+Q y,
$$

defined over an open interval $I$ of regular points, where $P, Q \in C^{\infty}(I)$ and $P(t)>0, Q(t)>0$ for all $t \in I$.

Let $f \in C^{\infty}(I)$. We shall be concerned with the oscillations of the iterates $f(t)$, $(L f)(t),\left(L^{2} f\right)(t), \ldots$ for $t \in I$. Specifically, we consider the influence on $f$ of the signs and sign changes in $I$ of the iterates $\left(L^{k} f\right)(t), k=0,1,2, \ldots$ This is a question of classical interest and has been the subject of much investigation [2], [4], [6]-[9], [11], [12]. The purpose of the present paper is to extend the results of [2] and [4].

In [2], the spectral theory of regular Sturm-Liouville boundary-value problems was used to generalize D. V. Widder's [11] classical theorems on completely convex functions (those $f \in C^{\infty}[0,1]$ with $(-1)^{k} f^{(2 k)}(x)>0,0<x<1, k=0,1,2, \ldots$ ). Widder proved in 1940 that a completely convex function is necessarily the restriction to $[0,1]$ of an entire function of exponential type at most $\pi$, and it is well known [12] that $f$ is completely convex if and only if

$$
f(x)=C \sin \pi x+\sum_{k=0}^{\infty}\left[f^{(2 k)}(0) A_{k}(1-x)+f^{(2 k)}(1) A_{k}(x)\right]
$$

where $C>0$ is a constant and where $A_{k}(x)$ is the Lidstone polynomial of degree $2 k+1$. In [2] we considered a boundary-value problem

Received by the editors November 27, 1978.

AMS (MOS) subject classifications (1970). Primary 34B25; Secondary 42A60.

Key words and phrases. Eigenfunction expansion, iterates of operators, sign changes. 


$$
L y=\lambda y, \quad y(a)=y(b)=0,
$$

with $L$ given by (1.1), on a closed, finite interval $[a, b]$. Assume that (1.3) has eigenvalues $0<\mu_{0}<\mu_{1}<\cdots$ and eigenfunctions $u_{0}(x), u_{1}(x), \ldots$, with $u_{0}(x)$ $>0$, and define the sequence $\left\{q_{n}(x)\right\}$ by $L q_{0}=L q_{1}=0, L q_{n+2}=q_{n}, q_{n}(a)=q_{n}(b)$ $=0$ for $n>2$, and $q_{0}(a)=q_{1}(b)=1, q_{1}(a)=q_{0}(b)=0$. Call a function $f \in$ $C^{\infty}[a, b] L$-positive if $\left(L^{k} f\right)(x) \geqslant 0, a<x \leqslant b, k=0,1,2, \ldots$ The principal result of [2] is the following.

THEOREM. A necessary and sufficient condition that $f$ be $L$-positive is that

$$
f(x)=C u_{0}(x)+\sum_{k=0}^{\infty}\left[\left(L^{k} f\right)(a) q_{2 k}(x)+\left(L^{k} f\right)(b) q_{2 k+1}(x)\right]
$$

where $C>0$ is a constant.

When $P=1, Q=0$ and $[a, b]=[0,1]$, the $L$-positive functions are just the completely convex functions, $u_{0}(x)=\sin \pi x$, and (1.4) reduces to (1.2).

The present paper is motivated partly by the question of what happens to the representation (1.4) if the problem (1.3) becomes singular at an endpoint.

In [4], Hille considered a more general boundary-value problem

$$
L y=\lambda y, \quad B_{a} y=B_{b} y=0,
$$

where $L$ (not necessarily selfadjoint) is given by $L y=P_{2}(x) y^{\prime \prime}+P_{1}(x) y^{\prime}+$ $P_{0}(x) y$, and where $B_{a}$ and $B_{b}$ are certain boundary forms corresponding to the endpoints $a$ and $b$. The problem (1.5) may be regular or singular, and $-\infty<a<b$ $<+\infty$, but it is assumed that (1.5) has a real, discrete spectrum of eigenvalues $0<\nu_{0}<\nu_{1}<\ldots$, and eigenfunctions $Y_{0}(x), Y_{1}(x), \ldots$ such that each $Y_{k}(x)$ has exactly $k$ sign changes in $(a, b), k=0,1,2, \ldots$ Various other "normalizing" type assumptions are made. Now a finite sum of eigenfunctions

$$
Y(t)=\sum_{j=0}^{N} C_{j} Y_{j}(t), \quad C_{N} \neq 0,
$$

has the property that its successive iterates

$$
\left(L^{m} Y\right)(t)=\sum_{j=0}^{N} C_{j} \nu_{j}^{m} Y_{j}(t)
$$

will have exactly $N$ sign changes in $(a, b)$ for all $m$ sufficiently large. It is natural to ask whether a function $f$ whose iterates $\left(L^{m} f\right)(t)$ have this property is necessarily of the form (1.6). To this end, Hille introduces a class $F$ of "admissible" functions $f$ which satisfy $f \in C^{\infty}(a, b)$ and

$$
B_{a}\left(L^{k} f\right)=B_{b}\left(L^{k} f\right)=0, \quad k=0,1,2, \ldots,
$$

the endpoint conditions being the same that determine the $Y_{k}$. If (1.5) is singular, the hypothesis $L^{k} f \in L^{2}(a, b), k=0,1,2, \ldots$, is also required. Finally, let $F_{N}$ be the subclass of $F$ consisting of all $f \in F$ such that each of $\left(L^{m} f\right)(t)$ has exactly $N$ sign changes in $(a, b)$ for all $m$ sufficiently large. Then the following holds [4]. 
TheORem (Hille). A necessary and sufficient condition that $f \in F_{N}$ is that

$$
f(t)=\sum_{j=0}^{N} C_{j} Y_{j}(t)
$$

where the $C_{j}$ are constant and $C_{N} \neq 0$.

In the present paper we give a representation which includes the version of (1.8) pertinent to our hypotheses as a special case, and which yields the analogy of (1.4) for singular problems. We do not obtain a direct generalization of Hille's theorem, in the sense of linearly weaker hypotheses; we give an example to show why such an extension is not possible.

For simplicity, we take the basic interval to be $\left[0, b^{*}\right)$, where $0<b^{*}<\infty$, there being no difficulty in extending our regime to other intervals. We shall suppose that $L$, given by (1.1), is regular at each $t \in\left[0, b^{*}\right)$ but is singular at $b^{*}$. We consider a boundary-value problem

$$
L y=\lambda y, \quad y(0)=0, \quad B^{*}(y)=0,
$$

where $B^{*}$, defined below, is a boundary form associated with the singular endpoint $b^{*}$. We assume that (1.9) has a discrete spectrum, $0<\lambda_{0}<\lambda_{1}<\ldots$, with eigenfunctions $\left\{y_{k}\right\}_{0}^{\infty}$ such that each $y_{k}$ has exactly $k$ sign changes in $\left(0, b^{*}\right)$, $k=0,1,2, \ldots$ The representation we derive has the form

$$
f(t)=\sum_{k=0}^{\infty}\left[\left(L^{k} f\right)(0) p_{2 k}(t)+B^{*}\left(L^{k} f\right) p_{2 k+1}(t)\right]+\sum_{j=0}^{N} c_{j} y_{j}(t),
$$

where the sequence $\left\{p_{k}\right\}$ is defined analogously to the $\left\{q_{k}\right\}$ of [2], and where the last nonvanishing $c_{j}$ reflects the frequency of oscillation of $\left(L^{m} f\right)(t)$, for $m$ large. On $\left[0, b^{*}\right)$ the conditions $(1.7)$ become

$$
\left(L^{k} f\right)(0)=B^{*}\left(L^{k} f\right)=0, \quad k=0,1,2, \ldots,
$$

and in this case (1.10) reduces to (1.8). In the case of $L$-positive functions on $\left[0, b^{*}\right)$ we show $N=0$ in (1.10), thus yielding the analogy of (1.4).

2. Preliminaries. We require certain general information about singular boundary-value problems, most of which can be found in [3]. Using the notation of [3], let $\lambda$ be a complex parameter and define the functions $\psi(t, \lambda)$ and $\varphi(t, \lambda)$ by

$$
\begin{aligned}
L \psi & =\lambda \psi, \quad L \varphi=\lambda \varphi, \\
\psi(0, \lambda) & =P(0) \varphi^{\prime}(0, \lambda)=0, \\
\varphi(0, \lambda) & =P(0) \psi^{\prime}(0, \lambda)=1 .
\end{aligned}
$$

Then $\psi$ and $\varphi$ are entire functions of $\lambda$. Let $m(\lambda)$ be the function defined by $m(\lambda)=\lim _{t \rightarrow b^{*}}\{-\varphi(t, \lambda) / \psi(t, \lambda)\}$. We will suppose that $m(\lambda)$ is meromorphic in the $\lambda$-plane with real, simple poles $\lambda_{0}, \lambda_{1}, \lambda_{2}, \ldots$ where $0<\lambda_{0}<\lambda_{1}<\ldots$ Then $S=\left\{\lambda_{k}\right\}_{0}^{\infty}$ is the spectrum of one of two possible types of boundary-value problems on $\left[0, b^{*}\right)$, corresponding to the limit-point or limit-circle cases.

For each $\lambda \notin S$, the function $\chi(t, \lambda)=\varphi(t, \lambda)+m(\lambda) \psi(t, \lambda)$ satisfies $\chi(0, \lambda)=$ $1, P(0) \chi^{\prime}(0, \lambda)=m(\lambda)$, and $\chi(t, \lambda) \in L^{2}\left[0, b^{*}\right)$. Thus for all $\lambda \notin S$ there is always at least one nontrivial solution to $L y=\lambda y$ of class $L^{2}\left[0, b^{*}\right)$. If $\chi$ is the only 
independent solution in $L^{2}\left[0, b^{*}\right)$, then $L$ is said to be of limit-point type at $b^{*}$. Otherwise, every solution to $L y=\lambda y$, for every $\lambda$, is in $L^{2}\left[0, b^{*}\right)$, and $L$ is said to be of limit-circle type at $b^{*}$. If the limit-point case prevails, the problem

$$
L y=\lambda y, \quad y(0)=0, \quad y \in L^{2}\left[0, b^{*}\right)
$$

is selfadjoint and has spectrum $S$. That is, $(L f, g)=(f, L g)$ whenever $f, g, L f, L g$ $\in L^{2}\left[0, b^{*}\right)$ and $f(0)=g(0)=0$, and the only nontrivial solutions to (2.2) are the multiples of $\psi\left(t, \lambda_{k}\right), k=0,1,2, \ldots$ In the limit-circle case, a boundary condition at $b^{*}$ must be introduced. First recall Green's identity

$$
\int_{\alpha}^{\beta}(g L f-f L g) d t=[f g](\beta)-[f g](\alpha),
$$

where $[f g](t)=P(t)\left[f(t) g^{\prime}(t)-f^{\prime}(t) g(t)\right]$. Recalling $0 \notin S$, put $\chi(t)=\chi(t, 0)$ into (2.3) in place of $f$, take $\alpha=0, \beta=b^{*}$ and let $L g \in L^{2}\left[0, b^{*}\right)$. There results

$$
-\int_{0}^{b^{*}} x L g d t=[x g]\left(b^{*}\right)-[x g](0) \text {. }
$$

In particular, the limit $[x g]\left(b^{*}\right)$ exists whenever $L g \in L^{2}\left[0, b^{*}\right)$, and this turns out to be the necessary expression for a boundary condition at $b^{*}$. Thus we define $B^{*}(y)=[x y]\left(b^{*}\right)=\lim _{t \rightarrow b^{*}}[x y](t)$, whenever the limit exists. Then [3] the problem

$$
L y=\lambda y, \quad y(0)=0, \quad B^{*}(y)=0
$$

is selfadjoint, in that $(L f, g)=(f, L g)$ when $f, L f, g, L g \in L^{2}\left[0, b^{*}\right)$ and $f(0)=g(0)$ $=B^{*}(f)=B^{*}(g)=0$. Again, the spectrum of (2.5) is $S$, and the nontrivial solutions to (2.5) are the multiples of $\psi\left(t, \lambda_{k}\right), k=0,1,2, \ldots$

An example of the limit-point case is Airy's equation on $[0, \infty)$; i.e., the boundary-value problem

$$
-y^{\prime \prime}+x y=\lambda y, \quad y(0)=0,
$$

is of limit-point type at $\infty$. Its eigenvalues are the zeros of the Airy function $\operatorname{Ai}(-x)$ (see [1], $x=2.338,4.087,5.521, \ldots$ approximately). The problem associated with Legendre's equation on $[0,1)$,

$$
-\left[\left(1-x^{2}\right) y^{\prime}\right]^{\prime}=\lambda y, \quad y(0)=0, \quad \lim _{x \rightarrow 1}\left(1-x^{2}\right) y^{\prime}(x)=0,
$$

is of limit-circle type at $b^{*}=1$ and has $S=\{2,12,30, \ldots\}$.

It so happens (Lemma 2.2) that in the limit-point case, the condition $B^{*}(y)=0$ is automatically satisfied if $y, L y \in L^{2}\left[0, b^{*}\right)$. Therefore, condition (2.5) together with $y \in L^{2}\left[0, b^{*}\right)$ serve to define the eigenfunctions for either the limit-point or limit-circle case.

We denote the normalized eigenfunctions by $y_{k}(t)$,

$$
y_{k}(t)=\frac{\psi\left(t, \lambda_{k}\right)}{\left\{\int_{0}^{b^{*}} \psi^{2}\left(\tau, \lambda_{k}\right) d \tau\right\}^{\frac{1}{2}}}, \quad k=0,1,2, \ldots
$$

Then the eigenfunction expansion associated with either problem has the form $f(t)=\sum_{k=0}^{\infty}\left(f, y_{k}\right) y_{k}(t)$, and this is valid for all $f \in L^{2}\left[0, b^{*}\right)$ sufficiently smooth (say $f \in C^{\infty}\left[0, b^{*}\right)$ ) with $f(0)=B^{*}(f)=0$. The convergence is absolute and uniform on compact subsets of $\left[0, b^{*}\right)$ (see [10]). We will also need the summability 
condition

$$
\sum_{k=0}^{\infty} \lambda_{k}^{-2}<\infty
$$

Our last hypothesis pertains to the limit-circle case only. If $L$ is of limit-circle type at $b^{*}$ we will suppose that

$$
\lambda_{k}^{-1}\left|y_{k}(t)\right|<K_{0}(k+1)^{-p}, \quad k=0,1,2, \ldots, \quad 0<t<b^{*},
$$

where $K_{0}>0$ is a constant and $p>1$, and that

$$
y_{0}(t)>\gamma_{0}>0, \quad t \text { sufficiently near } b^{*},
$$

where $\gamma_{0}$ is a constant. If $b^{*}=\infty$ the latter condition is assumed to hold for all $t$ sufficiently large. These hypotheses are patterned after the Legendre polynomials which arise in example (2.6). One can verify [1, Chapter 8] for this example that

$$
\lambda_{k}^{-} y_{k}(t)=\frac{(-1)^{k} P_{2 k+1}(t)}{(2 k+1)(2 k+2)}\left[\frac{\pi^{2}\left(2 k+\frac{1}{2}\right)}{\pi^{2}+\Psi^{\prime}(2 k+2)}\right]^{1 / 2}, \quad k=0,1,2, \ldots,
$$

where $\Psi(x)$ is the logarithmic derivative of the gamma function. Recalling $P_{2 k+1}(1)$ $=1,(\mathrm{lc}-1)$ and (lc-2) follow easily.

Now consider the functions $\psi, \varphi, x$. First note that the function $\psi(t)=\psi(t, 0)$ can be substituted for $g$ in (2.4) even if $\psi \notin L^{2}\left[0, b^{*}\right)$. Then

$$
B^{*}(\psi)=[x \psi]\left(b^{*}\right)=[x \psi](t)=[x \psi](0)=1, \quad 0<t<b^{*} .
$$

Also, trivially,

$$
[\chi \chi]\left(b^{*}\right)=B^{*}(\chi)=0 .
$$

If we put $f(t)=\psi(t, \lambda)$ and $g=\varphi(t, \lambda)$ into (2.3) with $\alpha=0$ and $\beta=T>0$, we get

$$
0=[\psi \varphi](T)-[\psi \varphi](0), \quad T>0,
$$

or

$$
[\psi \varphi](T)=[\psi \varphi](0)=-1
$$

Then

$$
\frac{d}{d t}\left(\frac{\varphi(t, \lambda)}{\psi(t, \lambda)}\right)=\frac{[\psi \varphi](t)}{P(t) \psi^{2}(t, \lambda)}=\frac{-1}{P(t) \psi^{2}(t, \lambda)},
$$

and so the quotient $\varphi(t, \lambda) / \psi(t, \lambda)$ is decreasing on $\left(0, b^{*}\right)$ wherever it is defined. Note that solutions to $L y=\lambda y$ also satisfy the fundamental identity

$$
\left.P y y^{\prime}\right|_{\alpha} ^{\beta}=\int_{\alpha}^{\beta}\left\{P\left(y^{\prime}\right)^{2}+(Q-\lambda) y^{2}\right\} d t
$$

If $\lambda<0$, our hypotheses on $P$ and $Q$ imply that $\psi(t, \lambda)$ and $\varphi(t, \lambda)$ are positive on $\left(0, b^{*}\right)$ with $\psi(t, \lambda)$ increasing. Thus the quotient $\{-\varphi(t, \lambda) / \psi(t, \lambda)\}$ increases through negative values to $m(\lambda)$ as $t \rightarrow b^{*}$. That is

$$
-\frac{\varphi(t, \lambda)}{\psi(t, \lambda)}<m(\lambda)^{\cdot}<0, \quad \lambda<0
$$


and so the function $\chi(t)=\chi(t, 0)=\varphi(t, 0)+m(0) \psi(t, 0)$ satisfies

$$
\chi(t)>0, \quad 0<t<b^{*} \text {. }
$$

In the following lemma and subsequently throughout this paper, all functions considered are assumed to be of continuity class $C^{\infty}\left[0, b^{*}\right)$.

LEMMA 2.1. Let $f$ satisfy $f(t)>0$ and $(L f)(t)>0$ on $\left[0, b^{*}\right)$. Then

$$
0<f(t)-f(0) \chi(t)<[\chi f](0) \psi(t)
$$

and

$$
0<f^{\prime}(t)-f(0) \chi^{\prime}(t)<[\chi f](0) \psi^{\prime}(t), \quad 0<t<b^{*} .
$$

Proof. Consider a subinterval $[a, b]$, where $0<a<b<b^{*}$. Define

$$
\chi_{b}(t)=\varphi(t)-\frac{\varphi(b)}{\psi(b)} \psi(t), \quad \psi_{b}(t)=\frac{\psi(t)}{\psi(b)} .
$$

Here, $\varphi(t)=\varphi(t, 0)$ and $\psi(t)=\psi(t, 0)$. Note that $\chi_{b}(t) \rightarrow \chi(t)=\chi(t, 0)$ as $b \rightarrow b^{*}$. Let

$$
\begin{gathered}
p_{0}(t ; a, b)=\chi_{b}(t) / \chi_{b}(a), \\
p_{1}(t ; a, b)=\frac{\psi(t)-\psi(a) p_{0}(t ; a, b)}{\psi(b)}, \quad a<t<b .
\end{gathered}
$$

Recall $\chi_{b}(b)=0, \psi(0)=0$ and that neither $\chi_{b}$ nor $\psi$ can have two zeros by (2.10). Then $L p_{0}=L p_{1}=0$ and $p_{0}(a ; a, b)=p_{1}(b ; a, b)=1$, and $p_{0}(b ; a, b)=p_{1}(a$; $a, b)=0$. The Green's function for $L$ on $[a, b]$ is given by

$$
G_{a b}(t, \tau)= \begin{cases}\frac{p_{0}(t ; a, b) p_{1}(\tau ; a, b)}{P(a) p_{1}^{\prime}(a ; a, b)}, & a<\tau<t<b, \\ \frac{p_{1}(t ; a, b) p_{0}(\tau ; a, b)}{P(a) p_{1}^{\prime}(a ; a, b)}, & a<t<\tau<b .\end{cases}
$$

The integral operator $\mathcal{G}_{a b}$ defined by

$$
\left(\mathcal{S}_{a b} h\right)(t)=\int_{a}^{b} G_{a b}(t, \tau) h(\tau) d \tau
$$

gives the unique solution to the problem $L y=h, y(a)=y(b)=0$. This leads to the identity

$$
f(t)=f(a) p_{0}(t ; a, b)+f(b) p_{1}(t ; a, b)+\left(\mathcal{G}_{a b} L f\right)(t),
$$

for $a<t<b$. Identity (2.10) shows that $p_{0}(t ; a, b)$ and $p_{1}(t ; a, b)$, thus $G_{a b}(t, \tau)$, are nonnegative in $[a, b]$. Hence $f(t)>0$ and $(L f)(t)>0$ imply

$$
f(t)>f(a) p_{0}(t ; a, b)
$$

and

$$
f(t)>f(b) p_{1}(t ; a, b), \quad 0<a<t<b<b^{*} .
$$

Letting $b \rightarrow b^{*}$ in (2.16) yields $f(t)>f(a) \chi(t) / \chi(a), a<t$. Therefore the quotient $f / x$ is increasing on $\left(0, b^{*}\right)$. In particular,

$$
f(t) / \chi(t)>f(0) / \chi(0)=f(0), \quad t>0,
$$


and this is the left side of (2.12). But $0 \leqslant(f / \chi)^{\prime}=\left(\chi f^{\prime}-f \chi^{\prime}\right) x^{-2}$, and so

$$
f^{\prime}(t) \geqslant(f(t) / \chi(t)) \chi^{\prime}(t) \geqslant f(0) \chi^{\prime}(t)
$$

which is the left side of (2.13).

Now put $g(t)=f(t)-f(0) \chi(t)$. Then $g(0)=0, g(t)>0$ and $(L g)(t)=(L f)(t)>0$. To complete the proof it suffices to show

$$
g(t) \leqslant P(0) g^{\prime}(0) \psi(t) \text { and } g^{\prime}(t)<P(0) g^{\prime}(0) \psi^{\prime}(t) .
$$

If we put $g$ into (2.17) and let $a \rightarrow 0$ we obtain

$$
g(t) \geqslant g(b) \psi(t) / \psi(b), \quad 0 \leqslant t<b .
$$

Letting $t \rightarrow 0$ gives $\left(P(0) \psi^{\prime}(0)=1\right)$

$$
g(b) / \psi(b)<P(0) g^{\prime}(0) \text {. }
$$

Since $b<b^{*}$ is arbitrary, this proves the first of (2.18). By (2.19), $g / \psi$ is decreasing on $\left(0, b^{*}\right)$ and so $0>(g / \psi)^{\prime}=\left(\psi g^{\prime}-g \psi^{\prime}\right) \psi^{-2}$, or

$$
g^{\prime}(t) \leqslant(g(t) / \psi(t)) \psi^{\prime}(t)<P(0) g^{\prime}(0) \psi^{\prime}(t) .
$$

This completes the proof of (2.18), thus the lemma.

The lemma can be thought of as a minimum-maximum principle: positive solutions of the differential inequality $L f>0$ lie between two distinguished solutions of $L f=0$.

Corollary 2.1. Suppose that $\left(L^{k} f\right)(t)>0, k=0,1,2, \ldots, 0<t<b^{*}$. Then

$$
0 \leqslant\left(L^{k} f\right)(t)-\left(L^{k} f\right)(0) \chi(t)<\left[\chi\left(L^{k} f\right)\right](0) \psi(t),
$$

and

$$
0<\left(L^{k} f\right)^{\prime}(t)-\left(L^{k} f\right)(0) \chi^{\prime}(t) \leqslant\left[\chi\left(L^{k} f\right)\right](0) \psi^{\prime}(t), \quad k=0,1,2, \ldots
$$

In the limit-circle case, $\chi$ and $\psi$ belong to $L^{2}\left[0, b^{*}\right)$ and so the same must be true of $f$ in (2.12).

Corollary 2.2. Suppose $L$ is of limit-circle type at $b^{*}$. Then $f(t)>0$ and $(L f)(t)>0$ imply $f \in L^{2}\left[0, b^{*}\right)$. If $\left(L^{k} f\right)(t)>0, k=0,1,2, \ldots$, then $L^{k} f \in$ $L^{2}\left[0, b^{*}\right), k=0,1,2, \ldots$

LEMMA 2.2. Suppose $L$ is of limit-point type at $b^{*}$ and let $f, L f \in L^{2}\left[0, b^{*}\right)$. Then $B^{*}(f)=0$. If $L^{k} f \in L^{2}\left[0, b^{*}\right), k=0,1,2, \ldots$ then $B^{*}\left(L^{k} f\right)=0, k=0,1,2, \ldots$

Proof. Let $a \rightarrow 0$ and $b \rightarrow b^{*}$ in (2.14) to obtain the Green's function for $L$ on $\left[0, b^{*}\right)$,

$$
G(t, \tau)= \begin{cases}\chi(t) \psi(\tau), & 0<\tau<t<b^{*}, \\ \psi(t) \chi(\tau), & 0 \leqslant t<\tau<b^{*}\end{cases}
$$

The corresponding integral operator is

$$
\begin{aligned}
(\mathcal{S} h)(t) & =\int_{0}^{b^{*}} G(t, \tau) h(\tau) d \tau \\
& =\chi(t) \int_{0}^{t} \psi(\tau) h(\tau) d \tau+\psi(t) \int_{t}^{b^{*}} \chi(\tau) h(\tau) d \tau
\end{aligned}
$$


This is defined for $h \in L^{2}\left[0, b^{*}\right)$. By writing out expressions for the pertinent derivatives, one can show that

$$
L \mathscr{G} h=h, \quad B^{*}(\mathcal{S} h)=0, \quad(\mathscr{S} h)(0)=0 .
$$

Moreover

$$
\mathcal{G} h \in L^{2}\left[0, b^{*}\right) \text { when } h \in L^{2}\left[0, b^{*}\right) .
$$

From the identity $L(f-\mathcal{G} L f)=0$ (or by letting $a \rightarrow 0, b \rightarrow b^{*}$ in (2.15)) we obtain $f=c_{1} \chi+c_{2} \psi+\mathcal{G} L f$ for certain constants $c_{1}$ and $c_{2}$. Since $\chi(0)=1$ and $\psi(0)=0$ we have in view of (2.8), (2.9) and (2.20),

$$
f(t)=f(0) \chi(t)+B^{*}(f) \psi(t)+(\mathcal{S} L f)(t) .
$$

By (2.21), each term in this equation belongs to $L^{2}\left[0, b^{*}\right)$ except $\psi(t)$. Hence $B^{*}(f)=0$.

LEMMA 2.3. Let $f(t)>0$ and $(L f)(t)>0$. Then the limits $B^{*}(f)$ and $\lim _{t \rightarrow b^{+}}[f(t) / \psi(t)]$ exist and are equal,

$$
0<B^{*}(f)=\lim _{t \rightarrow b^{*}}[f(t) / \psi(t)]<\infty .
$$

Proof. First we show $\chi^{\prime}(t)<0,0<t<b^{*}$. If $\chi^{\prime}\left(t_{0}\right)>0$ for some fixed $t_{0}>0$, then $\chi_{b}^{\prime}\left(t_{0}\right)>0$ for all $b$ sufficiently near $b^{*}$. Since $\chi_{b}(0)=1$ and $\chi_{b}(b)=0$ we must have $\chi_{b}^{\prime}\left(t_{1}\right)<0$ for some $t_{1} \in[0, b)$. Then $\chi_{b}^{\prime}(t)$ must vanish at some point $t \neq b$. However, (2.10) implies that $\chi_{b}(t) \chi_{b}^{\prime}(t)$ can vanish at most once in $\left[0, b^{*}\right)$, and this contradiction proves $\chi^{\prime}(t)<0, t>0$.

Multiply (2.13) by $P(t) \chi(t)$, multiply (2.12) by $-P(t) \chi^{\prime}(t)$, and add the resulting inequality. There results

$$
0<[x f](t)<[x f](0)[x \psi](t)=[x f](0) .
$$

Next, observe that $d[\chi f](t) / d t=-\chi(t)(L f)(t)<0$, so the function $[\chi f](t)$ is decreasing and positive. Therefore $0<B^{*}(f)<\infty$. Therefore, by (2.4) the improper integral

$$
0<\int_{0}^{b^{*}} x L f d \tau<\infty
$$

for $f$ satisfying the hypotheses of this lemma.

If $a=0,(2.15)$ becomes

$$
\begin{aligned}
f(t)= & f(0) \chi_{b}(t)+\frac{f(b)}{\psi(b)} \psi(t) \\
& +\chi_{b}(t) \int_{0}^{t} \psi L f d \tau+\psi(t) \int_{t}^{b} \chi_{b} L f d \tau .
\end{aligned}
$$

Since all terms are positive,

$$
f(t)>(f(b) / \psi(b)) \psi(t), \quad 0<t<b .
$$

This says that the quotient $(f / \psi)$ is decreasing on $\left(0, b^{*}\right)$, and therefore that the limit $c=\lim _{b \rightarrow b^{*}}[f(b) / \psi(b)]$ exists. Noting (2.24), we can allow $b \rightarrow b^{*}$ in (2.25) 
and use the Dominated Convergence Theorem to yield

$$
\begin{aligned}
f(t)= & f(0) \chi(t)+c \psi(t) \\
& +\chi(t) \int_{0}^{t} \psi L f d \tau+\psi(t) \int_{t}^{b^{*}} \chi L f d \tau .
\end{aligned}
$$

This expression is valid even though we do not assume $L f \in L^{2}\left[0, b^{*}\right)$. Equation (2.26) can be differentiated to give

$$
\begin{aligned}
f^{\prime}(t)= & f(0) \chi^{\prime}(t)+c \psi^{\prime}(t) \\
& +\chi^{\prime}(t) \int_{0}^{t} \psi L f d \tau+\psi^{\prime}(t) \int_{t}^{b^{*}} \chi L f d \tau .
\end{aligned}
$$

Multiply (2.26) by $P(t) \chi^{\prime}(t),(2.27)$ by $-P(t) \chi(t)$, and add the results. We find in view of (2.8) that

$$
[\chi f](t)=c+\int_{t}^{b^{*}} \chi L f d \tau
$$

Letting $t \rightarrow b^{*}$ gives the desired result.

Since $\chi(t)$ satisfis the hypothesis of the lemma (by (2.11)) we have

Corollary 2.3. $\lim _{t \rightarrow b^{*}}[\chi(t) / \psi(t)]=0$.

The following shows that the conditions $f(t)>0,(L f)(t)>0$ may be replaced by $L f \in L^{2}\left[0, b^{*}\right)$ in Lemma 2.3 .

Corollary 2.4. Let $L f \in L^{2}\left[0, b^{*}\right)$. Then $B^{*}(f)=\lim _{t \rightarrow b^{*}}[f(t) / \psi(t)]$.

Proof. Start with (2.25). This time the integral in (2.24) is finite by the Cauchy-Schwarz inequality. Proceed by the Dominated Convergence Theorem to (2.26), and from there to (2.28). This gives $c=B^{*}(f)$. Now divide (2.26) by $\psi(t)$, note Corollary 2.3 and let $t \rightarrow b^{*}$. There follows $c=\lim _{t \rightarrow b^{*}}[f(t) / \psi(t)]$, which completes the proof.

COROllary 2.5. If $L$ is of limit-point type at $b^{*}$ and $f, L f \in L^{2}\left[0, b^{*}\right)$, then $\lim _{t \rightarrow b^{*}}[f(t) / \psi(t)]=0$.

Proof. Use Lemma 2.2 and Corollary 2.4.

Corollary 2.6. If $h \in L^{2}\left[0, b^{*}\right)$, then $\lim _{t \rightarrow b^{*}}[(\mathcal{G} h)(t) / \psi(t)]=0$.

Proof. This follows from (2.20) and Corollary 2.4 .

We will need to consider limits of quotients $f(t) / \varphi(t, \lambda)$ for $\lambda<0$.

LEMMA 2.4. Let $f$ satisfy $\lim _{t \rightarrow b^{*}}[f(t) / \psi(t)]=0$. Then for $\lambda<0$ and $|\lambda|$ sufficiently large, $\lim _{t \rightarrow b^{*}}[f(t) / \varphi(t, \lambda)]=0$.

Proof. Put $\varphi_{\lambda}(t)=\varphi(t, \lambda)$. By Green's identity,

$$
\begin{aligned}
(\lambda-\mu) \int_{0}^{x} \varphi_{\mu} \varphi_{\lambda} d t & =\int_{0}^{x}\left(\varphi_{\mu} L \varphi_{\lambda}-\varphi_{\lambda} L \varphi_{\mu}\right) d t \\
& =\left.\left[\varphi_{\lambda} \varphi_{\mu}\right]\right|_{0} ^{x}=\left.P\left(\varphi_{\lambda} \varphi_{\mu}^{\prime}-\varphi_{\lambda}^{\prime} \varphi_{\mu}\right)\right|_{0} ^{x} \\
& =P(x) \varphi_{\lambda}^{2}(x)\left(\varphi_{\mu} / \varphi_{\lambda}\right)^{\prime}(x) .
\end{aligned}
$$


We have already noted that $\varphi_{\lambda}(t)$ and $\psi(t, \lambda)$ are positive for $\lambda<0$. Thus if $\mu<\lambda<0,\left(\varphi_{\mu} / \varphi_{\lambda}\right)^{\prime}(x) \geqslant 0$, so $\left(\varphi_{\lambda} / \varphi_{\mu}\right)$ is decreasing. Since $\left(\varphi_{\lambda} / \varphi_{\mu}\right)(0)=1$, we have $\varphi_{\lambda}(x) \leqslant \varphi_{\mu}(x), x \geqslant 0$. Hence $\varphi_{\lambda}(x)$ is decreasing as a function of $\lambda$ in the range $\lambda<0$. Consequently,

$$
\int_{0}^{x} \varphi_{\lambda} \psi d t \geqslant \int_{0}^{x} \varphi_{0} \psi d t, \quad \lambda \leqslant 0
$$

Another application of Green's identity gives

$$
\begin{aligned}
-\lambda \int_{0}^{x} \varphi_{\lambda} \psi d t & =-\int_{0}^{x} \psi L \varphi_{\lambda} d t \\
& =\left.\left[\psi \varphi_{\lambda}\right]\right|_{0} ^{x}=P(x) \psi^{2}(x)\left(\varphi_{\lambda} / \psi\right)^{\prime}(x)+1
\end{aligned}
$$

Using (2.29), we have

$$
P(x) \psi^{2}(x)\left(\varphi_{\lambda} / \psi\right)^{\prime}(x)>-1-\lambda \int_{0}^{x} \varphi_{0} \psi d t,
$$

and it follows that $\left(\varphi_{\lambda} / \psi\right)^{\prime}(x)>0$ for large enough $x, \lambda<0$, and $|\lambda|$ sufficiently large. For such $\lambda,\left(\psi / \varphi_{\lambda}\right)(x)$ is eventually decreasing, therefore its limit at $b^{*}$ exists.

Writing

$$
f(t) / \varphi_{\lambda}(t)=(f(t) / \psi(t))\left(\psi(t) / \varphi_{\lambda}(t)\right)
$$

the assertion of the lemma follows readily.

The relevance of Lemma 2.4 is found in the identity

$$
(L-\lambda) f=-\frac{1}{\varphi_{\lambda}} \frac{d}{d t}\left\{P \varphi_{\lambda}^{2} \frac{d}{d t}\left(\frac{f}{\varphi_{\lambda}}\right)\right\} .
$$

If $f$ satisfies the boundary conditions $f(0)=0$ and $[f(t) / \psi(t)] \rightarrow 0$ as $t \rightarrow b^{*}$, then $\left(f / \varphi_{\lambda}\right)$ vanishes at $t=0$ and $t=b^{*}$, for $\lambda \leqslant 0$ and $|\lambda|$ sufficiently large, by the lemma. Two applications of Rolle's Theorem in (2.30) show that each sign change of $f(t)$ in $\left(0, b^{*}\right)$ produces at least one sign change of $(L-\lambda) f(t)$ in $\left(0, b^{*}\right)$. (A sign change of $f$ at $x_{0}$ means that $f\left(x_{0}\right)=0$, that there is a $\delta>0$ such that $f$ does not vanish in either $\left(x_{0}-\delta, x_{0}\right)$ or $\left(x_{0}, x_{0}+\delta\right)$, and that $f(t)$ has opposite signs in the two half-intervals.) In Hille's terminology, the operator $L-\lambda$ is oscillation preserving with respect to the class of all $f$ such that $f(0)=0$ and $\left(f / \varphi_{\lambda}\right) \rightarrow 0, t \rightarrow b^{*}$, for all $\lambda$ large and negative.

Recall that the eigenfunctions of (2.5) are positive multiples of $\psi\left(t, \lambda_{k}\right)$. We are assuming that each $y_{k}(t)$ has exactly $k$ sign changes in $\left(0, b^{*}\right), k=0,1,2, \ldots$

LEMMA 2.5. For each nonnegative integer $k$ there exists a constant $\gamma_{k}>0$ such that

$$
\left|y_{k}(t) / y_{0}(t)\right|>\gamma_{k}
$$

for all $t$ sufficiently near $b^{*}$ (sufficiently large if $\left.b^{*}=\infty\right)$.

Proof. For each $k>0$

$$
\begin{aligned}
\left(\lambda_{0}-\lambda_{k}\right) \int_{0}^{x} y_{0} y_{k} d t & =\int_{0}^{x}\left(y_{k} L y_{0}-y_{0} L y_{k}\right) d t=\left.\left[y_{0} y_{k}\right]\right|_{0} ^{x} \\
& =P(x) y_{0}^{2}(x)\left(y_{k} / y_{0}\right)^{\prime}(x) .
\end{aligned}
$$


Since $y_{k}(x)$ has only $k$ sign changes in $\left(0, b^{*}\right)$, it is ultimately of constant sign as $x$ increases. If $y_{k}(x) \geqslant 0$ for all large $x$ (near $b^{*}$ ) then the left side of (2.32) is eventually decreasing. It tends to 0 by orthogonality. Thus $\left(y_{k} / y_{0}\right)^{\prime}(x)>0$, so that $\left(y_{k} / y_{0}\right)$ is increasing (and positive) for all $x$ near $b^{*}$, whence (2.31). On the other hand, if $y_{k}(x) \leqslant 0$ for all $x$ near $b^{*}$ then the left side of (2.32) is eventually increasing to 0 . That is, $\left(y_{k} / y_{0}\right)^{\prime}(x) \leqslant 0$, so $\left(y_{k} / y_{0}\right)$ is decreasing for $x$ near $b^{*}$. Therefore $\left|y_{k} / y_{0}\right|$ is increasing, implying that (2.31) holds in this case also.

3. Series expansions. Recall that all functions $f$ considered are assumed to be of class $C^{\infty}\left[0, b^{*}\right)$. Suppose $L^{k} f \in L^{2}\left[0, b^{*}\right), k=0,1,2, \ldots$ Define $p_{0}(t)=\chi(t)$ and $p_{1}(t)=\psi(t)$. Then (2.22) becomes

$$
f(t)=f(0) p_{0}(t)+B^{*}(f) p_{1}(t)+(\mathcal{G} L f)(t)
$$

in the limit-circle case, and

$$
f(t)=f(0) p_{0}(t)+(\mathcal{G} L f)(t)
$$

in the limit-point case (Lemma 2.2). Define $p_{2 k}(t)=\left(\mathcal{G}^{k} p_{0}\right)(t), k=0,1,2, \ldots$, and if $L$ is of limit-circle type at $b^{*}$ let $p_{2 k+1}(t)=\left(\mathcal{S}^{k} p_{1}\right)(t), k=0,1,2, \ldots$ Thus the sequence $\left\{p_{k}\right\}$ satisfies

$$
L p_{0}=L p_{1}=0, \quad L p_{k+2}=p_{k}, \quad p_{k+2}=\mathcal{S}_{p_{k}}, \quad k=0,1,2, \ldots
$$

By (2.20) and the definitions of $p_{0}$ and $p_{1}$,

$$
\begin{gathered}
p_{0}(0)=1, \quad p_{1}(0)=0, \quad B^{*}\left(p_{0}\right)=0, \quad B^{*}\left(p_{1}\right)=1, \\
p_{k}(0)=B^{*}\left(p_{k}\right)=0, \quad k=2,3,4, \ldots
\end{gathered}
$$

The $p_{2 k+1}$ remains undefined in the limit-point case.

Replace $f$ by $L f$ in (3.1) and (3.2), apply the operator $\mathcal{G}$ to the resulting equations and substitute the expressions for $\mathcal{G} L f$ back into (3.1) and (3.2). This gives

$$
f(t)=f(0) p_{0}(t)+B^{*}(f) p_{1}(t)+(L f)(0) p_{2}(t)+B^{*}(L f) p_{3}(t)+\left(\mathcal{G}^{2} L^{2} f\right)(t)
$$

or

$$
f(t)=f(0) p_{0}(t)+(L f)(0) p_{2}(t)+\left(\mathcal{G}^{2} L^{2} f\right)(t)
$$

according as $L$ is of limit-circle or limit-point type at $b^{*}$. Continuing this procedure, we are led to the identities

$$
f(t)=\sum_{k=0}^{m-1}\left[\left(L^{k} f\right)(0) p_{2 k}(t)+B^{*}\left(L^{k} f\right) p_{2 k+1}(t)\right]+\left(\mathcal{G}^{m} L^{m} f\right)(t)
$$

or

$$
f(t)=\sum_{k=0}^{m-1}\left(L^{k} f\right)(0) p_{2 k}(t)+\left(\mathcal{G}^{m} L^{m} f\right)(t),
$$

depending on which case prevails. To economize notation we adopt a convention which will combine (3.5) and (3.6). If the limit-point case holds at $b^{*}$ we define $p_{2 k+1}(t)=0$ for $k=1,2,3, \ldots$ We already have $B^{*}\left(L^{k} f\right)=0$, if $L^{k_{f}} \in$ $L^{2}\left[0, b^{*}\right)$, by Lemma 2.2. With this interpretation, (3.6) is subsumed by (3.5). 
THEOREM 3.1. Let $L^{k} f \in L^{2}\left[0, b^{*}\right), k=0,1,2, \ldots$ and suppose $\lim _{k \rightarrow \infty} \lambda_{0}^{-k}\left\|L^{k} f\right\|_{2}=0$. Then

$$
f(x)=\sum_{k=0}^{\infty}\left[\left(L^{k} f\right)(0) p_{2 k}(x)+B^{*}\left(L^{k} f\right) p_{2 k+1}(x)\right]
$$

with uniform convergence on compact subsets of $\left[0, b^{*}\right)$.

Proof. We apply the expansion $f=\Sigma_{0}^{\infty}\left(f, y_{k}\right) y_{k}$ to a typical remainder term $\mathcal{G}^{m} L^{m} f$ in (3.5). By selfadjointness, $\left(\mathcal{G}^{m} L^{m} f, y_{k}\right)=\lambda_{k}^{-m}\left(L^{m} f, y_{k}\right)$, so we have

$$
\left(\mathcal{G}^{m} L^{m} f\right)(x)=\sum_{k=0}^{\infty} \lambda_{k}^{-m}\left(L^{m} f, y_{k}\right) y_{k}(x)
$$

with uniform convergence on compact subsets. Note that

$$
\lambda_{k}^{-1} y_{k}(x)=\left(\mathcal{G} y_{k}\right)(x)=\int_{0}^{b^{*}} G(x, t) y_{k}(t) d t
$$

so the Cauchy-Schwarz inequality gives

$$
\lambda_{k}^{-1}\left|y_{k}(x)\right| \leqslant\left\{\int_{0}^{b^{*}} G^{2}(x, t) d t\right\}^{\frac{1}{2}} \text {. }
$$

Considering the definition of $G$, it follows that the sequence $\left\{\lambda_{k}^{-1} y_{k}(x)\right\}$ is uniformly bounded on compact subsets. Thus for any compact set $K \subseteq\left[0, b^{*}\right)$ there is a constant $M>0$ such that

$$
\left|\left(\mathcal{G}^{m} L^{m} f\right)(x)\right| \leqslant M \sum_{k=0}^{\infty} \lambda_{k}^{-m+1}\left|\left(L^{m} f, y_{k}\right)\right|, \quad x \in K,
$$

when $m$ is large enough for the series to converge. By hypothesis, $\lambda_{0}^{-m}\left(L^{m} f, y_{k}\right)=$ $\varepsilon_{m}$, where $\varepsilon_{m} \rightarrow 0$ as $m \rightarrow \infty$. Hence

$$
\left|\left(\mathcal{G}^{m} L^{m} f\right)(x)\right| \leqslant \lambda_{0} M \varepsilon_{m} \sum_{k=0}^{\infty}\left(\lambda_{0} / \lambda_{k}\right)^{m-1} \leqslant \lambda_{0} M \varepsilon_{m} \sum_{k=0}^{\infty}\left(\lambda_{0} / \lambda_{k}\right)^{2},
$$

for $m=3,4,5, \ldots$ and $x \in K$. Here we have used (2.7). Since the remainder tends to 0 , (3.7) holds.

If $\lambda$ is complex, $|\lambda|<\lambda_{0}$, then the function $\chi(t, \lambda)$ satisfies the hypotheses of Theorem 3.1. In the limit-circle case the same is true of the function $\psi_{\lambda}(t)=$ $\psi(t, \lambda)$. For these functions (3.7) becomes

$$
\chi(t, \lambda)=\sum_{k=0}^{\infty} p_{2 k}(t) \lambda^{k}, \quad \psi(t, \lambda)=\sum_{k=0}^{\infty} B^{*}\left(\psi_{\lambda}\right) p_{2 k+1}(t) \lambda^{k}
$$

We can view these as the power series expansions, in the variable $\lambda$, of $\chi(t, \lambda)$ and $\psi(t, \lambda)$. We have, for example, the property that $\left\{p_{2 k}(t) \lambda^{k}\right\}$ and $\left\{p_{2 k+1}(t) \lambda^{k}\right\}$ are uniformly bounded on compact sets. That is, given $\lambda$ complex, $|\lambda|<\lambda_{0}$, and a compact set $K \subseteq\left[0, b^{*}\right)$, there exists a constant $M>0$, depending only on $K$ and $\lambda$, such that

$$
\left|p_{2 k}(t) \lambda^{k}\right| \leqslant M, \quad\left|p_{2 k+1}(t) \lambda^{k}\right| \leqslant M, \quad t \in K, \quad k=0,1,2, \ldots
$$

The latter inequality trivially holds in the limit-point case since then $p_{2 k+1}(t)=0$ for $k=1,2,3, \ldots$. 
TheOREM 3.2. Let $f$ satisfy $L^{k} f \in L^{2}\left[0, b^{*}\right), k=0,1,2, \ldots$, and

$$
\sum_{k=0}^{\infty}\left|\lambda^{-k}\right|\left\{\left|\left(L^{k} f\right)(0)\right|+\left|B^{*}\left(L^{k} f\right)\right|\right\}<\infty
$$

for some $\lambda,|\lambda|<\lambda_{0}$. Then the series

$$
S(x)=\sum_{k=0}^{\infty}\left[\left(L^{k} f\right)(0) p_{2 k}(x)+B^{*}\left(L^{k} f\right) p_{2 k+1}(x)\right]
$$

converges absolutely and uniformly on compact subsets of $\left[0, b^{*}\right)$.

Proof. Express the terms in the series as

$$
\left(L^{k} f\right)(0) p_{2 k}(x)=\left\{\lambda^{-k}\left(L^{k} f\right)(0)\right\}\left\{\lambda^{k} p_{2 k}(x)\right\},
$$

and similarly for the term $B^{*}\left(L^{k} f\right) p_{2 k+1}(t)$. Now use (3.9), (3.10) and the Weierstrass $M$-test.

The series $S(x)$ does not necessarily converge to $f(x)$. For instance, if $f$ is taken to be an eigenfunction $y_{k}(x)$, then $S(x) \equiv 0$.

Definition. The class $c(\lambda), \lambda$ complex and $|\lambda|<\lambda_{0}$, consists of all functions $f$ such that $L^{k} f \in L^{2}\left[0, b^{*}\right), k=0,1,2, \ldots$, and such that (3.10) holds.

TheOREM 3.3. Let $f \in c(\lambda)$. Then

$$
f(x)=\sum_{k=0}^{\infty}\left[\left(L^{k} f\right)(0) p_{2 k}(x)+B^{*}\left(L^{k} f\right) p_{2 k+1}(x)\right]+R(x)
$$

with absolute and uniform convergence on compact subsets of $\left[0, b^{*}\right)$, where $R$ satisfies $L^{k} R \in L^{2}\left[0, b^{*}\right),\left(L^{k} R\right)(0)=0$, and $B^{*}\left(L^{k} R\right)=0, k=0,1,2, \ldots$

Proof. By identity (3.5) and Theorem 3.2, (3.12) holds with $R(x)=$ $\lim _{m \rightarrow \infty}\left(\mathcal{G}^{m} L^{m} f\right)(x)$. Thus there remains only to establish the properties of $R(x)$. Let $S(x)$ be given by (3.11) so that $f(x)=S(x)+R(x)$.

The first step is to show that the series

$$
\sum_{k=m}^{\infty}\left[\left(L^{k} f\right)(0) p_{2 k-2 m}(x)+B^{*}\left(L^{k} f\right) p_{2 k-2 m+1}(x)\right]
$$

represents an $L^{2}\left[0, b^{*}\right)$ function. It converges for all $x$ by (3.9) and (3.10). Now the operator norm of $\mathcal{G}$ is $\lambda_{0}^{-1}$ (see [5]); that is,

$$
\|\mathcal{G} f\|_{2}<\lambda_{0}^{-1}\|f\|_{2}, \quad f \in L^{2}\left[0, b^{*}\right) .
$$

Then $\left\|p_{2 k}\right\|_{2}=\left\|\mathcal{G}^{k} p_{0}\right\|_{2}<\lambda_{0}^{-k}\|x\|_{2}$ and $\left\|p_{2 k+1}\right\|_{2}<\lambda_{0}^{-k}\|\psi\|_{2}$ in the limit-circle case. Thus by the triangle inequality

$$
\begin{aligned}
\left\|\sum_{k=m}^{\infty}\left[\left(L^{k} f\right)(0) p_{2 k-2 m}+B^{*}\left(L^{k} f\right) p_{2 k-2 m+1}\right]\right\|_{2} \\
\leqslant \lambda_{0}^{m} \sum_{k=m}^{\infty} \lambda_{0}^{-k}\left\{\left|\left(L^{k} f\right)(0)\right|\|X\|_{2}+\left|B^{*}\left(L^{k} f\right)\right|\|\psi\|_{2}\right\}
\end{aligned}
$$

and this is finite by (3.10). In the limit-point case, the terms involving $\psi$ are omitted. The case $m=0$ shows $S \in L^{2}\left[0, b^{*}\right)$; thus $R \in L^{2}\left[0, b^{*}\right)$. 
We now show that the operator $L$ can be applied termwise to $S(x)$. Let $n$ be a positive integer. Then

$$
\begin{aligned}
\left|\sum_{k=1}^{n}\left[\left(L^{k} f\right)(0) G(x, t) p_{2 k-2}(t)+B^{*}\left(L^{k} f\right) G(x, t) p_{2 k-1}(t)\right]\right| \\
<\sum_{k=1}^{n}\left[\left|\left(L^{k} f\right)(0)\right| G(x, t) p_{2 k-2}(t)+\left|B^{*}\left(L^{k} f\right)\right| G(x, t) p_{2 k-1}(t)\right] \\
<\sum_{k=1}^{\infty}\left[\left|\left(L^{k} f\right)(0)\right| G(x, t) p_{2 k-2}(t)+\left|B^{*}\left(L^{k} f\right)\right| G(x, t) p_{2 k-1}(t)\right],
\end{aligned}
$$

recalling $G(x, t)>0$. The infinite series in (3.13) converges by (3.9) and (3.10). By the Monotone Convergence Theorem the infinite series in (3.13) can be integrated termwise with respect to $t$, resulting in

$$
\sum_{k=1}^{\infty}\left[\left|\left(L^{k} f\right)(0)\right| p_{2 k}(x)+\left|B^{*}\left(L^{k} f\right)\right| p_{2 k+1}(x)\right]
$$

by (3.3). Thus the first finite series in (3.13) represents a function $S_{n}(t)$, suppressing for the moment the variable $x$, which is dominated for all $n$ by an $L^{1}\left[0, b^{*}\right)$ function, namely the last series in (3.13). By the Dominated Convergence Theorem the corresponding infinite series can be integrated termwise, so that we have

$$
\begin{aligned}
\mathcal{G}\left(\sum_{k=1}^{\infty}\left[\left(L^{k} f\right)(0) p_{2 k-2}+B^{*}\left(L^{k} f\right) p_{2 k-1}\right]\right)(x) \\
=\sum_{k=1}^{\infty}\left[\left(L^{k} f\right)(0) p_{2 k}(x)+B^{*}\left(L^{k} f\right) p_{2 k+1}(x)\right] \\
=S(x)-f(0) p_{0}(x)-B^{*}(f) p_{1}(x), \quad 0<x<b^{*} .
\end{aligned}
$$

Apply the operator $L$ to both sides of this equation, note (2.20) and (3.3), and obtain

$$
(L S)(x)=\sum_{k=1}^{\infty}\left[\left(L^{k} f\right)(0) p_{2 k-2}(x)+B^{*}\left(L^{k} f\right) p_{2 k-1}(x)\right]
$$

The argument for the higher iterates is much the same, as is the proof that $L^{k} S \in L^{2}\left[0, b^{*}\right)$. Thus we have

$$
\left(L^{m} S\right)(x)=\sum_{k=m}^{\infty}\left[\left(L^{k} f\right)(0) p_{2 k-2 m}(x)+B^{*}\left(L^{k} f\right) p_{2 k-2 m+1}(x)\right]
$$

and

$$
\left\|L^{m} S\right\|_{2}<\lambda_{0}^{m} \sum_{k=m}^{\infty} \lambda_{0}^{-k}\left\{\left|\left(L^{k} f\right)(0)\right|\|x\|_{2}+\left|B^{*}\left(L^{k} f\right)\right|\|\psi\|_{2}\right\}
$$

The latter inequality shows $\lambda_{0}^{-m}\left\|L^{m} S\right\|_{2} \rightarrow 0$ as $m \rightarrow \infty$. 
Since $L^{m} R=L^{m} f-L^{m} S$, then $L^{m} R \in L^{2}\left[0, b^{*}\right)$. Putting $x=0$ in (3.14) and using (3.4) shows $\left(L^{m} R\right)(0)=0, m=0,1,2, \ldots$ There remains only to show $B^{*}\left(L^{m} R\right)=0$.

It follows from the proof of Lemma 2.3 that $\left[x p_{2 k}\right](b)>0$ and $\left[x p_{2 k+1}\right](b)>0$ for $0<b<b^{*}$. Moreover,

$$
\frac{d}{d t}\left[\chi p_{2 k}\right](t)=-\chi(t) p_{2 k-2}(t)<0
$$

and similarly for $p_{2 k+1}$. Then.

$$
0<\left[x p_{2 k}\right](b)<\left[x p_{2 k}\right](0)=P(0) p_{2 k}^{\prime}(0), \quad k=1,2,3, \ldots
$$

Also

$$
P(0) p_{2 k}^{\prime}(0)=\int_{0}^{b^{*}} \chi(\tau)\left(\mathcal{G}^{k-1} \chi\right)(\tau) d \tau<\lambda_{0}^{-k+1}\|\chi\|_{2}^{2}
$$

and putting this together with (3.15) we find $0<\lambda_{0}^{k}\left[x p_{2 k}\right](b)<M$, where $M$ is some constant. A similar result holds in the limit-circle case for $p_{2 k+1}$. Then for each integer $m$,

$$
\begin{array}{r}
\left|\sum_{k=m}^{\infty}\left(L^{k} f\right)(0)\left[X p_{2 k}\right](b)+B^{*}\left(L^{k} f\right)\left[X p_{2 k+1}\right](b)\right| \\
<M \sum_{k=m}^{\infty} \lambda_{0}^{-k}\left\{\left|\left(L^{k} f\right)(0)\right|+\left|B^{*}\left(L^{k} f\right)\right|\right\}
\end{array}
$$

and this tends to 0 as $m \rightarrow \infty$. Therefore the infinite series

$$
\sum_{k=0}^{\infty}\left[\left(L^{k} f\right)(0)\left[X p_{2 k}\right](b)+B^{*}\left(L^{k} f\right)\left[X p_{2 k+1}\right](b)\right]
$$

converges uniformly in the variable $b$. The proof that it converges to $[x S](b)$ amounts to showing that the series for $S(x)$ can be differentiated termwise. From $p_{2 k}=\mathcal{S}_{p_{2 k-2}}$ we have

$$
p_{2 k}^{\prime}(x)=\int_{0}^{b^{*}} \frac{\partial}{\partial x} G(x, t) p_{2 k-2}(t) d t
$$

and the Cauchy-Schwarz inequality yields

$$
\left|p_{2 k}^{\prime}(x)\right|<M\left\|p_{2 k-2}\right\|_{2}<M \lambda_{0}^{-k+1}\|x\|_{2}
$$

where $x$ ranges over a compact set $K$ and $M$ depends only on $K$. In particular the sequence $\left\{\lambda_{0}^{k} p_{2 k}^{\prime}(x)\right\}$ is uniformly bounded on compact sets, and the same can be said for $\left\{\lambda_{0}^{k} p_{2 k+1}^{\prime}(x)\right\}$. Using (3.10) one can now easily show $S^{\prime}=\sum_{k=0}^{\infty}\left[\left(L^{k} f\right)(0) p_{2 k}^{\prime}\right.$ $\left.+B^{*}\left(L^{k} f\right) p_{2 k+1}^{\prime}\right]$. We conclude that (3.16) equals $[\chi S](b)$. By uniform convergence 
of (3.16), we can let $b \rightarrow b^{*}$ and use (3.4) to get $B^{*}(R)=B^{*}(f)-B^{*}(S)=0$. The proof for higher iterates $L^{k} R$ is the same. This completes the proof.

Corollary 3.1. Let $f \in c(\lambda)$ and let $S(x)$ be given by (3.11). Then $\lim _{m \rightarrow \infty} \lambda^{-m}\left(L^{m} S\right)(x)=0$ and $\lim _{m \rightarrow \infty} \lambda^{-m}\left(L^{m} S\right)^{\prime}(x)=0$, uniformly on compact subsets of $\left[0, b^{*}\right)$.

Proof. By (3.14) and the method of the preceding proof, we have for $j=0,1$,

$$
\begin{aligned}
\left|\lambda^{-m}\left(L^{m} S\right)^{j}(x)\right| & =\left|\lambda-m \sum_{k=m}^{\infty}\left[\left(L^{k} f\right)(0) p_{2 k-2 m}^{(j)}(x)+B^{*}\left(L^{k} f\right) p_{2 k-2 m+1}^{(j)}(x)\right]\right| \\
& <\sum_{k=m}^{\infty}\left[| \lambda | ^ { - k } \left\{\left|\left(L^{k} f\right)(0)\right|\left|\lambda^{k-m_{p}(j)-2 m}(x)\right|\right.\right. \\
& \left.\left.+\left|B^{*}\left(L^{k} f\right)\right|\left|\lambda^{k-m_{p}(j)-2 m+1}(x)\right|\right\}\right] \\
& <M \sum_{k=m}^{\infty}|\lambda|^{-k}\left\{\left|\left(L^{k} f\right)(0)\right|+\left|B^{*}\left(L^{k} f\right)\right|\right\},
\end{aligned}
$$

where $M>0$ is a constant. This expression tends to 0 as $m \rightarrow \infty$.

There will be occasion to apply the operators $L$ and $B^{*}$ termwise to certain eigenfunction series $\Sigma c_{k} y_{k}$. If $f \in L^{2}\left[0, b^{*}\right)$ it has a formal expansion $f \sim$ $\sum_{k=0}^{\infty}\left(f, y_{k}\right) y_{k}$. Since $\mathcal{G} f \in L^{2}\left[0, b^{*}\right)$ and $(\mathcal{G} f)(0)=B^{*}(\mathcal{G} f)=0$ we have $\left(\mathcal{G} f, y_{k}\right)=$ $\lambda_{k}^{-1}\left(\mathcal{G} f, L y_{k}\right)=\lambda_{k}^{-1}\left(L \mathcal{G} f, y_{k}\right)=\lambda_{k}^{-1}\left(f, y_{k}\right)$. Therefore $(\mathcal{G} f)(x)=$ $\sum_{k=0}^{\infty} \lambda_{k}^{-1}\left(f, y_{k}\right) y_{k}(x)$ with absolute and uniform convergence on compact subsets of $\left[0, b^{*}\right)$.

Let $f \in L^{2}\left[0, b^{*}\right)$ be such that the series $f_{1}(x)=\sum_{k=0}^{\infty} \lambda_{k}\left(f, y_{k}\right) y_{k}(x)$ converges absolutely and uniformly on compact sets. Then $f_{1} \in L^{2}\left[0, b^{*}\right), \mathcal{G} f_{1}=f$, and $f(0)=B^{*}(f)=0$. Moreover, $L f=L\left(\mathcal{G} f_{1}\right)=f_{1}$ by (2.20). These remarks establish the following.

LEMMA 3.1. Let $f \in L^{2}\left[0, b^{*}\right)$ have an absolutely convergent representation $f(x)=$ $\sum_{k=0}^{\infty}\left(f, y_{k}\right) y_{k}(x)$, uniformly on compact sets, and suppose that the series $\sum_{k=0}^{\infty} \lambda_{k}\left(f, y_{k}\right) y_{k}(x)$ converges absolutely and uniformly on compact sets. Then $f(0)=$ $B^{*}(f)=0$ and $(L f)(x)=\sum_{k=0}^{\infty} \lambda_{k}\left(f, y_{k}\right) y_{k}(x)$.

LEMMA 3.2. Let $0<\lambda<\lambda_{0}$ and let $\chi_{\lambda}(t)=\chi(t, \lambda), \psi_{\lambda}(t)=\psi(t, \lambda), w_{\lambda}(t)=$ $\chi_{\lambda}(t)-\chi(t)$, and $\sigma_{\lambda}(t)=\psi_{\lambda}(t)-B^{*}\left(\psi_{\lambda}\right) \psi(t)$. Then the functions $\left[w_{\lambda}(t) / y_{0}(t)\right]$ and, in the limit-circle case, $\left[\sigma_{\lambda}(t) / y_{0}(t)\right]$ are bounded on $\left(0, b^{*}\right)$.

Proof. First observe that

$$
\begin{aligned}
\left(p_{0}, y_{0}\right) & =\left(\chi, y_{0}\right)=\int_{0}^{b^{*}} x y_{0} d \tau=\lambda_{0}^{-1} \int_{0}^{b^{*}} x L y_{0} d \tau \\
& =\lambda_{0}^{-1}\left\{\left[y_{0} x\right]\left(b^{*}\right)-\left[y_{0} x\right](0)\right\}=\lambda_{0}^{-1}\left[x y_{0}\right](0)=\lambda_{0}^{-1} P(0) y_{0}^{\prime}(0)
\end{aligned}
$$


Then for fixed $t$,

$$
\begin{aligned}
P(t) \chi_{\lambda}^{2}(t)\left(y_{0} / \chi_{\lambda}\right)^{\prime}(t) & =\left[\chi_{\lambda} y_{0}\right](t)=\left[\chi_{\lambda} y_{0}\right](0)+\int_{0}^{t}\left[y_{0} L \chi_{\lambda}-\chi_{\lambda} L y_{0}\right] d \tau \\
& =P(0) y_{0}^{\prime}(0)+\left(\lambda-\lambda_{0}\right) \int_{0}^{t} \chi_{\lambda} y_{0} d \tau \\
& =P(0) y_{0}^{\prime}(0)+\left(\lambda-\lambda_{0}\right) \int_{0}^{t}\left(\sum_{k=0}^{\infty} p_{2 k} \lambda^{k}\right) y_{0} d \tau \\
& =P(0) y_{0}^{\prime}(0)+\left(\lambda-\lambda_{0}\right) \sum_{k=0}^{\infty}\left(\int_{0}^{t} p_{2 k} y_{0} d \tau\right) \lambda^{k} \\
& \geqslant P(0) y_{0}^{\prime}(0)+\left(\lambda-\lambda_{0}\right) \sum_{k=0}^{\infty}\left(p_{2 k}, y_{0}\right) \lambda^{k} \\
& =P(0) y_{0}^{\prime}(0)+\left(\lambda-\lambda_{0}\right)\left(p_{0}, y_{0}\right) \sum_{k=0}^{\infty}\left(\lambda / \lambda_{0}\right)^{k} \\
& =P(0) y_{0}^{\prime}(0)+\left(\lambda-\lambda_{0}\right)\left(p_{0}, y_{0}\right)\left(1-\lambda \lambda_{0}^{-1}\right)^{-1} \\
& =P(0) y_{0}^{\prime}(0)-\lambda_{0}\left(p_{0}, y_{0}\right)=0 .
\end{aligned}
$$

This implies $\left(y_{0} / \chi_{\lambda}\right)^{\prime}(t) \geqslant 0$, and so $\left[\chi_{\lambda}(t) / y_{0}(t)\right]$ must be decreasing on $\left(0, b^{*}\right)$. Thus it has a limit as $t \rightarrow b^{*}$, so the same must be true for $\left[\chi(t) / y_{0}(t)\right]$ since $0<\chi(t)<\chi_{\lambda}(t)$. Hence $\left[w_{\lambda}(t) / y_{0}(t)\right]$ is bounded on intervals $\left[\varepsilon, b^{*}\right), \varepsilon>0$. By (3.8), $w_{\lambda}(t)=\sum_{k=1}^{\infty} p_{2 k}(t) \lambda^{k}$. It follows from (3.17) that this series can be differentiated termwise, and there results

$$
\left|w_{\lambda}^{\prime}(0)\right|<M \lambda_{0}\|x\|_{2} \sum_{k=1}^{\infty}\left(\lambda / \lambda_{0}\right)^{k}
$$

Since $y_{0}^{\prime}(0) \neq 0$, L'Hospital's rule implies that $\left[w_{\lambda}(t) / y_{0}(t)\right]$ is bounded in neighborhoods of $t=0$. This completes the proof for $w_{\lambda}(t)$.

Now suppose the limit-circle case holds. The eigenfunction expansions for the functions $p_{2 m+1}(t)$ are $p_{2 m+1}(t)=\sum_{k=0}^{\infty} \lambda_{k}^{-m}\left(p_{1}, y_{k}\right) y_{k}(t), m=0,1,2, \ldots$ By (lc-1) the function $p_{3}(t)$ is bounded on $\left(0, b^{*}\right)$, indeed

$$
\left|p_{3}(t)\right|<\sum_{k=0}^{\infty}\left|\left(p_{1}, y_{k}\right) \lambda_{k}^{-1} y_{k}(t)\right| \leqslant K_{0}\left\|p_{1}\right\|_{2} \sum_{k=0}^{\infty}(k+1)^{-p} \text {. }
$$

If $m>2$, use (lc-1) again to find

$$
\begin{aligned}
\left|\lambda_{0}^{m} p_{2 m+1}(t)\right| & <\lambda_{0} \sum_{k=0}^{\infty}\left|\left(p_{1}, y_{k}\right)\left(\lambda_{0} / \lambda_{k}\right)^{m-1} \lambda_{k}^{-1} y_{k}(t)\right| \\
& <\lambda_{0}\left\|p_{1}\right\|_{2} K_{0} \sum_{k=0}^{\infty}\left(\lambda_{0} / \lambda_{k}\right)^{m-1}(k+1)^{-p}
\end{aligned}
$$

It follows that the sequence $\left\{\lambda_{0}^{m} p_{2 m+1}(t)\right\}_{1}^{\infty}$ is uniformly bounded on $\left[0, b^{*}\right)$, say

$$
\left|\lambda_{0}^{m} p_{2 m+1}(t)\right|<K_{1}, \quad 0<t<b^{*}, \quad m=1,2,3, \ldots
$$


By (3.8), $\sigma_{\lambda}(t)=\sum_{k=1}^{\infty} B^{*}\left(\psi_{\lambda}\right) p_{2 k+1}(t) \lambda^{k}$, and so (3.18) gives

$$
\left|\sigma_{\lambda}(t)\right| \leqslant K_{1} B^{*}\left(\psi_{\lambda}\right) \sum_{k=1}^{\infty}\left(\lambda / \lambda_{0}\right)^{m}
$$

Therefore $\sigma_{\lambda}(t)$ is bounded on $\left[0, b^{*}\right)$. By (lc-2), $\left[\sigma_{\lambda}(t) / y_{0}(t)\right]$ is bounded on intervals $\left[\varepsilon, b^{*}\right), \varepsilon>0$. To finish the proof apply L'Hospital's rule, using a bound on $p_{2 k+1}^{\prime}(x)$ analogous to (3.17).

Definition. Let $N$ be a positive integer and let $0<\lambda<\lambda_{0}$. The class $c(\lambda, N)$ is the subclass of $c(\lambda)$ consisting of all $f \in c(\lambda)$ for which there exist disjoint, open, finite subintervals $J_{1}, J_{2}, \ldots, J_{N}$ of $\left(0, b^{*}\right)$ and constants $m_{1}>0, m_{2}>0$ such that:

(i) for all large $m,\left(L^{m} f\right)(t)$ has exactly one sign change in each of $J_{1}, J_{2}, \ldots, J_{N}$;

(ii) for all large $m$, we have

$$
\left|\frac{\left(L^{m} f\right)(t)-\left(L^{m_{f}}\right)(0) \chi(t)-B^{*}\left(L^{m} f\right) \psi(t)}{\lambda^{m} y_{0}(t)}\right|>m_{1}, \quad t \notin J_{1} \cup J_{2} \cup \cdots \cup J_{N} ;
$$

(iii) for all large $m$, we have

$$
\left|\frac{d}{d t}\left\{\lambda^{-m}\left(L^{m} f\right)(t)\right\}\right| \geqslant m_{2}, \quad t \in J_{1} \cup J_{2} \cup \cdots \cup J_{N} .
$$

For $N=0$ define $c(\lambda, 0)$ by dropping (i) and (iii) but requiring (ii) to hold everywhere in $\left(0, b^{*}\right)$.

We are now ready to prove the main result of this paper.

THeOREM 3.4. Let $f \in c(\lambda), 0<\lambda<\lambda_{0}$. Then a necessary and sufficient condition that $f \in c(\lambda, N)$ is that

$$
f(t)=\sum_{k=0}^{\infty}\left[\left(L^{k} f\right)(0) p_{2 k}(t)+B^{*}\left(L^{k} f\right) p_{2 k+1}(t)\right]+\sum_{j=0}^{N} c_{j} y_{j}(t),
$$

where $c_{N} \neq 0$.

Remarks. We are going to express, via Theorem $3.3, f(x)=S(x)+R(x)$, $L^{m} f=L^{m} S+L^{m} R$, where $\left(L^{m} R\right)(0)=B^{*}\left(L^{m} R\right)=0$, and then apply Hille's method to the function $R$. But first we have to have precise information about the sign changes of $L^{m} R$. Conditions (ii) and (iii) will insure that the sign changes of $L^{m} f$ will correspond one-to-one with those of $L^{m} R$ in $J_{1} \cup \cdots \cup J_{N}$. There may be other sign changes of $L^{m} f$ outside $J_{1} \cup \cdots \cup J_{N}$, so that the total number of oscillations of $L^{m} f$ may exceed $N$. Note, however, that if $\left(L^{m} f\right)(0)=B^{*}\left(L^{m} f\right)=0$ for all $m$, then (ii) implies that $L^{m} f$ does not have sign changes outside $J_{1}$ $\cup \cdots \cup J_{N}$. A direct generalization of Hille's theorem would say that if $L^{m} f$ has $N$ sign changes in $\left(0, b^{*}\right)$ for all large $m$ then (3.19) holds with the same $N$. Hence that type of extension is impossible. As an example, take the function $\chi_{b}\left(t, \lambda_{1}\right)=$ $\varphi\left(t, \lambda_{1}\right)-\left[\varphi\left(b, \lambda_{1}\right) / \psi\left(b, \lambda_{1}\right)\right] \psi\left(t, \lambda_{1}\right), 0<\lambda_{1}<\lambda_{0}$, and assume the limit-circle case holds. Here $\chi_{b}\left(b, \lambda_{1}\right)=0$, and this is the only sign change of $L^{m} \chi_{b}\left(t, \lambda_{1}\right)=$ $\lambda_{1}^{m} \chi_{b}\left(t, \lambda_{1}\right)$. By Theorem 3.1, $\chi_{b}\left(t, \lambda_{1}\right)$ is represented by its infinite series expansion in (3.19), so the eigenfunction part vanishes identically. 
Proof of THE THEOREM. Let $f \in c(\lambda, N), N>0$, and write via (3.12) $f(x)=$ $S(x)+R(x)$. Then $\lambda^{-m}\left(L^{m} f\right)^{(j)}(x)=\lambda^{-m}\left(L^{m} S\right)^{(j)}(x)+\lambda^{-m}\left(L^{m} R\right)^{(j)}(x)$ for $j=$ 0,1 and $m=0,1,2, \ldots$ By Corollary $3.1, \lambda^{-m}\left(L^{m} S\right)^{(j)}(x)$ tends to 0 uniformly on compact subsets of $\left[0, b^{*}\right)$. By condition (iii), $\left|\lambda^{-m}\left(L^{m} R\right)^{\prime}(x)\right|>m_{2} / 2$ (say) for $x \in J_{1} \cup J_{2} \cup \cdots \cup J_{N}$, and for all $m$ sufficiently large. Then in $J_{1} \cup \cdots \cup J_{N}$, for such $m, L^{m} R$ is monotone, so it can have at most one sign change in each of $J_{1}, \ldots, J_{N}$.

Rewrite the equation $L^{m} f=L^{m} S+L^{m} R$ as

$$
\begin{aligned}
\frac{\left(L^{m} f\right)(t)-\left(L^{m} f\right)(0) \chi(t)-B^{*}\left(L^{m} f\right) \psi(t)}{\lambda^{m} y_{0}(t)}=\frac{\left(L^{m} R\right)(t)}{\lambda^{m} y_{0}(t)} \\
+\sum_{k=1}^{\infty}\left[\frac{\left(L^{m+k} f\right)(0)}{\lambda^{m+k}} \frac{\lambda^{k} p_{2 k}(t)}{y_{0}(t)}+\frac{B^{*}\left(L^{m+k} f\right)}{\lambda^{m+k}} \frac{\lambda^{k} p_{2 k+1}(t)}{y_{0}(t)}\right] .
\end{aligned}
$$

By Lemma 3.2 and the representations of $\chi(t, \lambda)$ and $\psi(t, \lambda)$ by (3.8), we have

$$
\frac{\lambda^{k} p_{2 k}(t)}{y_{0}(t)} \leqslant M, \quad \frac{\lambda^{k} p_{2 k+1}(t)}{y_{0}(t)} \leqslant M, \quad 0 \leqslant t<b^{*}, \quad k=1,2,3, \ldots,
$$

where the constant $M$ depends only on $\lambda$. Then the infinite series in (3.20) is dominated by

$$
M \sum_{k=m+1}^{\infty} \lambda^{-k}\left\{\left|\left(L^{k} f\right)(0)\right|+\left|B^{*}\left(L^{k} f\right)\right|\right\}
$$

and this tends to 0 by (3.10). By (ii)

$$
\left|\frac{\left(L^{m} R\right)(t)}{\lambda^{m} y_{0}(t)}\right|>\frac{m_{1}}{2}, \quad t \notin J_{1} \cup \cdots \cup J_{N},
$$

for all $m$ sufficiently large. Therefore $L^{m} R$ can experience sign changes only in $J_{1} \cup \cdots \cup J_{N}$ if $m$ is large enough.

Let $J_{1}=\left(\alpha_{1}, \beta_{1}\right)$. The terms $\lambda^{-m}\left(L^{m} f\right)(0) \chi(t)\left[y_{0}(t)\right]^{-1}$ and $\lambda^{-m} B^{*}\left(L^{m} f\right) \psi(t)\left[y_{0}(t)\right]^{-1}$ each tend to $0, m \rightarrow \infty$, at $t=\alpha_{1}$ and $t=\beta_{1}$. By (ii),

$$
\left|\frac{\left(L^{m} f\right)(t)}{\lambda^{m} y_{0}(t)}\right|>\frac{m_{1}}{2}, \quad m \text { sufficiently large, }
$$

at $t=\alpha_{1}$ and $t=\beta_{1}$. Noting that the infinite series in (3.20) also tends to 0 at $t=\alpha_{1}$ and $t=\beta_{1}$, we find

$$
\frac{\left(L^{m} f\right)(t)-L^{m} R(t)}{\lambda^{m} y_{0}(t)} \rightarrow 0, \quad m \rightarrow \infty, \quad t=\alpha_{1} \text { and } t=\beta_{1} .
$$

Since $\left(L^{m} f\right)(t)$ has precisely one sign change in $J_{1}$ the signs of $\left(L^{m} f\right)(t)$ at $\alpha_{1}$ and $\beta_{1}$ have to be different. By the preceding estimates the same must be true of $\left(L^{m} R\right)(t)$. The same argument holds for $J_{2}, \ldots, J_{N}$. Consequently,

for $N>0,\left(L^{m} R\right)(t)$ has precisely one sign change in each of $J_{1}, J_{2}, \ldots, J_{N}$ and no other sign changes

for $m$ sufficiently large. 
If $N=0$, (ii) is required to hold for all $t$. Again one shows that the infinite series in (3.20) is small for large $m$. Thus $\lambda^{-m}\left(L^{m} R\right)(t)\left[y_{0}(t)\right]^{-1}$ is bounded away from 0 , and we have

$$
\begin{aligned}
& \text { for } N=0 \text { and } m \text { sufficiently large, } \\
& \left(L^{m} R\right)(t) \text { has no sign changes in }\left(0, b^{*}\right) .
\end{aligned}
$$

Now let $m_{0}$ be so large that (3.21) or (3.22) holds for $m \geqslant m_{0}$, and also sufficiently large that the conclusion of Lemma 2.4 holds with $\lambda=-\lambda_{m}, m>m_{0}$. That is, the operator $L+\lambda_{m}$ is oscillation-preserving with respect to the class of all $f$ such that $f(0)=B^{*}(f)=0$. At this point we could invoke Hille's theorem and conclude that $R$ is a finite sum of eigenfunctions. The argument is simple, however, and so we give the gist of it for completeness.

Introduce the function

$$
\Phi_{m, k}(t)=\sum_{n=0}^{\infty}\left\{\frac{4 \lambda_{m} \lambda_{n}}{\left(\lambda_{m}+\lambda_{n}\right)^{2}}\right\}^{k}\left(R, y_{n}\right) y_{n}(t),
$$

for nonnegative integers $m$ and $k$. The bracketed expression is at most 1 , and since $\left(L^{m} R\right)(0)=B^{*}\left(L^{m} R\right)=0$ for all $m$, we have

$$
\left|\left(R, y_{n}\right)\right|=\left|\lambda_{n}^{-p}\left(L^{p} R, y_{n}\right)\right| \leqslant \lambda_{n}^{-p}\left\|L^{p} R\right\|_{2} \text { for } p=0,1,2, \ldots
$$

By Lemma 3.1,

$$
\left(L^{p} \Phi_{m, k}\right)(t)=\sum_{n=0}^{\infty}\left\{\frac{4 \lambda_{m} \lambda_{n}}{\left(\lambda_{m}+\lambda_{n}\right)^{2}}\right\}^{k} \lambda_{n}^{p}\left(R, y_{n}\right) y_{n}(t)
$$

and

$$
\left(L^{p} \Phi_{m, k}\right)(0)=B^{*}\left(L^{p} \Phi_{m, k}\right)=0 \text { for all } p, m, k .
$$

Obviously, then,

$$
\left(\left(L+\lambda_{m}\right)^{p} \Phi_{m, k}\right)(0)=B^{*}\left(\left(L+\lambda_{m}\right)^{p} \Phi_{m, k}\right)=0
$$

for all $p, m, k$.

Let $V(f)$ denote the number of sign changes in $\left(0, b^{*}\right)$ of a given function $f$. Then by (3.23) and our remarks following (2.30), we have $V\left[\left(\left(L+\lambda_{m}\right)^{p} \Phi_{m, k}\right)\right]>V\left(\Phi_{m, k}\right)$, $m>m_{0}$. Note that

$$
\begin{aligned}
\left(\left(L+\lambda_{m}\right)^{2 k} \Phi_{m, k}\right)(t) & =\left(4 \lambda_{m}\right)^{k} \sum_{n=0}^{\infty} \lambda_{n}^{k}\left(R, y_{n}\right) y_{n}(t) \\
& =\left(4 \lambda_{m}\right)^{k}\left(L^{k} R\right)(t) .
\end{aligned}
$$

By (3.21) and (3.22), $V\left[\left(L+\lambda_{m}\right)^{2 k} \Phi_{m, k}\right]=N, k>m_{0}, m>m_{0}$, and so

$$
V\left(\Phi_{m, k}\right)<N, \quad k>m_{0}, \quad m>m_{0} .
$$

We will show that (3.24) is not possible unless $R$ reduces to a finite sum of eigenfunctions. 
If infinitely many coefficients $\left(R, y_{n}\right)$ do not vanish, we choose $m$ so that $m>m_{0}, m>N$ and $\left(R, y_{m}\right) \neq 0$. With this $m$, divide the series for $\Phi_{m k}$ as follows,

$$
\Phi_{m, k}(t)=\sum_{n \neq m}\left\{\frac{4 \lambda_{m} \lambda_{n}}{\left(\lambda_{m}+\lambda_{n}\right)^{2}}\right\}^{k}\left(R, y_{n}\right) y_{n}(t)+\left(R, y_{m}\right) y_{m}(t) .
$$

Let $\delta_{m}=\max _{n \neq m}\left[\left(4 \lambda_{m} \lambda_{n}\right) /\left(\lambda_{m}+\lambda_{n}\right)^{2}\right]$, so that

$$
\left|\Phi_{m, k}(t)-\left(R, y_{m}\right) y_{m}(t)\right| \leqslant \sum_{n \neq m} \delta_{m}^{k}\left|\left(R, y_{n}\right) y_{n}(t)\right| \text {. }
$$

Let $[\varepsilon, b]$ be a compact subset of $\left(0, b^{*}\right)$ containing all the sign changes of $y_{m}(t)$. There is a constant $T$ such that $\sum_{n=0}^{\infty}\left|\left(R, y_{n}\right) y_{n}(t)\right|<T$ for all $t \in[\varepsilon, b]$. Thus on $[\varepsilon, b]$

$$
\left|\Phi_{m, k}(t)-\left(R, y_{m}\right) y_{m}(t)\right| \leqslant T \delta_{m}^{k}, \quad k \geqslant m_{0}, \quad m \geqslant m_{0}, \quad m>N .
$$

Since $\delta_{m}<1, \delta_{m}^{k} \rightarrow 0$ as $k \rightarrow \infty$. Pick $k$ so large that each sign change of $y_{m}(t)$ in $[\varepsilon, b]$ is accompanied by a sign change of $\Phi_{m, k}(t)$. As $V\left[y_{m}\right]=m>N$, we have contradicted (3.24). This proves that $\left(R, y_{n}\right)=0$ for all $n$ sufficiently large, that is

$$
R(t)=\sum_{n=0}^{N_{1}} c_{n} y_{n}(t), \quad N_{1}<\infty
$$

Since $V\left[L^{m} R\right]=N, m$ large, it is easy to see that $N_{1}=N$. This completes the proof of sufficiency.

Let $f \in c(\lambda)$ and suppose (3.19) holds. Then

$$
\lambda^{-m}\left(L^{m} f\right)^{(i)}(t)=\lambda^{-m}\left(L^{m} S\right)^{(i)}(t)+\sum_{j=0}^{N} c_{j}\left(\lambda_{j} / \lambda\right)^{m} y_{j}^{(i)}(t)
$$

for $i=0,1$ and $m=0,1,2, \ldots$ Choose finite, open, disjoint intervals $J_{1}, J_{2}, \ldots, J_{N}$ and a constant $m_{3}>0$ such that $\left|y_{N}^{\prime}(t)\right|>m_{3}$ for $t \in J_{1}$ $\cup \cdots \cup J_{N}$, and such that $y_{N}$ has exactly one sign change in each of $J_{1}, \ldots, J_{N}$. The infinite series part of (3.25) tends uniformly to 0 on $J_{1} \cup \cdots \cup J_{N}, m \rightarrow \infty$, by Corollary 3.1. Since $c_{N} \neq 0$ and $0<\lambda<\lambda_{0}<\cdots<\lambda_{N}$, the term in the sum in (3.25) corresponding to $j=N$ will become more dominant as $m \rightarrow \infty$. Thus it is clear that (i) and (iii) hold for sufficiently large $m$.

Now write

$$
\begin{array}{r}
\left|\frac{\left(L^{m} f\right)(t)-\left(L^{m} f\right)(0) \chi(t)-B^{*}\left(L^{m} f\right) \psi(t)}{\lambda^{m} y_{0}(t)}-\sum_{j=0}^{N} c_{j}\left(\lambda_{j} / \lambda\right)^{m}\left[y_{j}(t) / y_{0}(t)\right]\right| \\
<\sum_{k=1}^{\infty}\left\{\left|\frac{\left(L^{m+k} f\right)(0)}{\lambda^{m+k}}\right| \frac{\lambda^{k} p_{2 k}(t)}{y_{0}(t)}+\left|\frac{B^{*}\left(L^{m+k} f\right)}{\lambda^{m+k}}\right| \frac{\lambda^{k} p_{2 k+1}(t)}{y_{0}(t)}\right\} .
\end{array}
$$

Arguing as before (the discussion below (3.20)) the infinite series in (3.26) tends to 0 uniformly on $\left[0, b^{*}\right)$ as $m \rightarrow \infty$. Write the $j$-sum in (3.26) as

$$
\left(\lambda_{N} / \lambda\right)^{m} c_{N}\left(y_{N} / y_{0}\right) \sum_{j=0}^{N}\left(c_{j} / c_{N}\right)\left(\lambda_{j} / \lambda_{N}\right)^{m}\left(y_{j} / y_{N}\right)
$$


By Lemma $2.5\left|y_{N} / y_{0}\right|$ is increasing for all $t$ sufficiently large. Arguing as in Lemma 2.5, one can also show that $\left|y_{j} / y_{N}\right|$ is bounded for all $t$ sufficiently near $b^{*}$. Clearly $\left(y_{j} / y_{0}\right) \rightarrow\left(y_{j}^{\prime}(0) / y_{0}^{\prime}(0)\right)$ as $t \rightarrow 0$. Hence the $j$-sum is bounded away from 0 off of $J_{1} \cup \cdots \cup J_{N}$. This proves (ii).

For functions with positive iterates, the constraints imposed by the class $c(\lambda, N)$ can be avoided.

Theorem 3.5. Let $f$ satisfy $L^{k} f \in L^{2}\left[0, b^{*}\right), k=0,1,2, \ldots$ Then a necessary and sufficient condition that $\left(L^{k} f\right)(t) \geqslant 0, k=0,1,2, \ldots, 0<t<b^{*}$, is that $\left(L^{k} f\right)(0)>0, B^{*}\left(L^{k} f\right)>0$, for all $k$, and

$$
f(t)=\sum_{k=0}^{\infty}\left[\left(L^{k} f\right)(0) p_{2 k}(t)+B^{*}\left(L^{k} f\right) p_{2 k+1}(t)\right]+c_{0} y_{0}(t)
$$

where $c_{0} \geqslant 0$.

Proof. For the sufficiency it is enough to note that the operator $L$ can be applied termwise to (3.27). We omit the details of the argument.

By (2.23) all terms in (3.5) are nonnegative. We let $m \rightarrow \infty$ to obtain

$$
f(t)=\sum_{k=0}^{\infty}\left[\left(L^{k} f\right)(0) p_{2 k}(t)+B^{*}\left(L^{k} f\right) p_{2 k+1}(t)\right]+R(t) .
$$

Using positivity and arguing as in Theorem 3.3 we can show that

$$
\left(L^{m} f\right)(t)=\sum_{k=0}^{\infty}\left[\left(L^{m+k} f\right)(0) p_{2 k}(t)+B^{*}\left(L^{m+k} f\right) p_{2 k+1}(t)\right]+\left(L^{m} R\right)(t),
$$

$m=0,1,2, \ldots$ In particular, using the one term involving $p_{1}(t)=\psi(t)$ leads to

$$
0 \leqslant\left(\left(L^{m} R\right)(t)\right) / \psi(t) \leqslant\left(\left(L^{m} f\right)(t)\right) / \psi(t)-B^{*}\left(L^{m} f\right) \text {. }
$$

Let $t \rightarrow b^{*}$ and use Lemma 2.3 to find $B^{*}\left(L^{m} R\right)=0$. Obviously $\left(L^{m} R\right)(0)=0$, $m=0,1,2, \ldots$.

Now pick up the argument of the preceding theorem at the definition of $\Phi_{m, k}(t)$ and put $N=0$. Unless $\left(R, y_{n}\right)=0$ for $n>0,\left(L^{m} R\right)(t)$ will have a sign change for all large $m$, at variance with (3.28).

\section{REFERENCES}

1. M. Abramowitz and I. A. Stegun (Eds.), Handbook of mathematical functions with formulas, graphs and mathematical tables, Nat. Bur. Standards Appl. Math. Ser., no. 55, Superintendent of Documents, U. S. Government Printing Office, Washington, D. C., 1964. MR 29 \#4914.

2. J. D. Buckholtz and J. K. Shaw, Generalized completely convex functions and Sturm-Liouville operators, SIAM J. Math. Anal. 6 (1975), 812-828.

3. E. A. Coddington and N. Levinson, Theory of ordinary differential equations, McGraw-Hill, New York, 1955.

4. E. Hille, On the oscillation of differential transforms. II, Trans. Amer. Math. Soc. 52 (1942), 463-497.

5. __ Lectures on ordinary differential equations, Addison-Wesley, Reading, Mass., 1969.

6. G. Polya and N. Wiener, On the oscillation of the derivatives of a periodic function, Trans. Amer. Math. Soc. 52 (1942), 249-256.

7. J. K. Shaw, Analytic properties of generalized completely convex functions, SIAM J. Math. Anal. 8 (1977), 271-279. 
8. J. K. Shaw and W. R. Winfrey, Positivity properties of linear differential operators, J. Math. Anal. Appl. 65 (1978), 184-200.

9. G. Szegö, On the oscillation of differential transforms. I, Trans. Amer. Math. Soc. 52 (1942), 450-462.

10. E. C. Titchmarsh, Eigenfunction expansions associated with second-order differential equations, Vol. I, Clarendon Press, Oxford, 1962.

11. D. V. Widder, Functions whose even derivatives have a prescribed sign, Proc. Nat. Acad. Sci. U.S.A. 26 (1940), 657-659.

12. 387-398.

Department of Mathematics, Virginia Polytechnic Institute and State University, BlacksBURG, VIRGINIA 24061 